\title{
TWO RESULTS ON THE WEIGHTED POINCARÉ INEQUALITY ON COMPLETE KÄHLER MANIFOLDS
}

\author{
Ovidiu Munteanu
}

\begin{abstract}
In this paper we consider complete noncompact Kähler manifolds $M^{m}$ that satisfy the weighted Poincaré inequality with a weight function $\rho(x)$ that has limit zero at infinity of $M$. We prove that if the Ricci curvature lower bound $\operatorname{Ric}_{M}(x) \geq-4 \rho(x)$ holds on $M$ then the manifold has one nonparabolic end and if the bisectional curvature is bounded from below by $B K_{M}(x) \geq-\frac{\rho(x)}{m^{2}}$ then the manifold has one end, thus it is connected at infinity. The two results that we prove are the Kähler version of Theorem 6.3 and Theorem 7.2 in [L-W4] and improve some results in [L-W].
\end{abstract}

\section{Introduction}

In [L-W4], P. Li and J. Wang studied the class of Riemannian manifolds that satisfy a weighted Poincaré inequality and have the Ricci curvature bounded from below in terms of the weight function. In particular, if the weight function is constant equal to the greatest lower bound of the spectrum of the Laplacian, the rigidity theorems proved in [L-W1] and [L-W2] for manifolds with positive spectrum are recovered. Let us recall that a Riemannian manifold $M^{n}$ of dimension $n$ is said to satisfy the weighted Poincaré inequality with a positive weight function $\rho(x)$ if

$$
\int_{M} \rho(x) \phi^{2}(x) d v \leq \int_{M}|\nabla \phi|^{2}(x) d v,
$$

for any compactly supported smooth function $\phi \in C_{c}^{\infty}(M)$.

Moreover, if $M$ is complete with respect to the $\rho$-metric defined by

$$
d s_{\rho}^{2}=\rho d s_{M}^{2},
$$

$M$ is said to satisfy the property $\left(P_{\rho}\right)$.

Corollary 1.4 in [L-W4] states that the weighted Poincaré inequality is equivalent to nonparabolicity of the manifold and this shows how large the class of manifolds satisfying $\left(P_{\rho}\right)$ is.

We are interested in the manifolds satisfying property $\left(P_{\rho}\right)$ for a weight function $\rho$ that has limit zero at infinity,

$$
\lim _{x \rightarrow \infty} \rho(x)=0 .
$$

In this case Li-Wang have proved that for $n \geq 3$, if

$$
\operatorname{Ric}_{M}(x) \geq-\frac{n-1}{n-2} \rho(x),
$$

Received by the editors November 7, 2006.

Reaserch partially supported by NSF grant No. DMS-0503735 
the manifold has only one nonparabolic end and furthermore if

$$
\operatorname{Ric}_{M}(x) \geq-\frac{4}{n-1} \rho(x),
$$

then the manifold has one end.

A natural question is to investigate the corresponding setting for Kähler manifolds. $\mathrm{Li}$ and Wang have proved in $[\mathrm{L}-\mathrm{W}]$ that if $M^{m}$ is a complete noncompact Kähler manifold of complex dimension $m \geq 2$ satisfying property $\left(P_{\rho}\right)$ with

$$
\lim _{x \rightarrow \infty} \rho(x)=0,
$$

and the Ricci curvature is bounded from below by

$$
\operatorname{Ric}_{M}(x) \geq-4 \rho(x)
$$

then $M$ has at most two nonparabolic ends. The result for parabolic ends states that if

$$
\lim _{x \rightarrow \infty} \rho(x)=0,
$$

and the bisectional curvature of $M$ admits the lower bound

$$
B K_{M}(x) \geq-\frac{1}{m^{2}} \rho(x)
$$

then $M$ has at most 2 ends for $m \geq 3$ and at most 4 ends if $m=2$. Note that the above assumption on the bisectional curvature means that with respect to any unitary frame,

$$
R_{\alpha \bar{\alpha} \beta \bar{\beta}}(x) \geq-\left(1+\delta_{\alpha \bar{\beta}}\right) \frac{1}{m^{2}} \rho(x) .
$$

To round off the picture in the Kähler setting, let us recall that when $\rho$ is constant, $\rho=\lambda_{1}(M)>0$, if the bisectional curvature lower bound $B K_{M} \geq-\frac{1}{m^{2}} \lambda_{1}(M)$ holds on $M$, then the manifold has at most two ends. Moreover, if the manifold has two ends and bounded curvature, then it is isometrically covered by $\mathbb{C H}^{m}$. In other words, all manifolds where equality holds in the Kähler version of Cheng's upper bound estimate for $\lambda_{1}(M)$ have the structure mentioned above ([L-W]).

However, if $\rho$ is not constant so far only in the case $\rho \rightarrow 0$ as $x \rightarrow \infty$ we are able to draw some information on the structure of $M$.

The purpose of this article is to improve the theorems of Li-Wang in the Kähler category, for $\rho \rightarrow 0$ at infinity of $M$. Our improvement consists of showing that $M$ needs to have one nonparabolic end if (1) holds and if (2) holds and $m \geq 3$ then $M$ has one end. Therefore, in this sense for Kähler manifolds we have analogous results to those for Riemannian manifolds.

Before discussing the results, let us make some observations. First, our argument does not work if $m=2$ and (2) holds. There is no indication yet that in this case the manifold needs to have only one end. Another remark concerns the bisectional curvature lower bound that is always assumed in the parabolic ends case mentioned above. Using a different argument, in [M] we generalized Li-Wang's result for manifolds with $\lambda_{1}(M)>0$ and admitting the corresponding Ricci curvature lower bound, Ric $_{M} \geq-\frac{2(m+1)}{m^{2}} \lambda_{1}(M)$. Similar results might hold for the weighted Poincaré inequality. 


\section{The results}

Theorem 1. Let $M^{m}$ be a complete Kähler manifold of complex dimension $m \geq 2$ and with property $\left(P_{\rho}\right)$. Suppose that the Ricci curvature of $M$ is bounded from below at any $x \in M$ by

$$
\operatorname{Ric}_{M}(x) \geq-4 \rho(x)
$$

If moreover

$$
\lim _{x \rightarrow \infty} \rho(x)=0
$$

then $M$ has only one nonparabolic end.

Proof. Let us assume by absurd that $M$ has at least two nonparabolic ends and thus by the theory of Li-Tam ([L-T]) there exists a bounded harmonic function with finite Dirichlet integral on $M$.

We may assume that $\inf f=0$ and $\sup f=1$ with infimum achieved at infinity of a nonparabolic end $E$ and supremum at infinity of the other nonparabolic end $F=M \backslash E$.

It is a fact that since $f$ is harmonic and with finite Dirichlet integral then $f$ is pluriharmonic and one has the improved Bochner formula for pluriharmonic functions:

$$
\Delta h \geq-2 \rho h+h^{-1}|\nabla h|^{2},
$$

where $h=|\nabla f|^{\frac{1}{2}}$. Moreover, Li-Wang [L-W] have shown that this is in fact an equality and concluded from here that the manifold must have at most two nonparabolic ends.

We will improve their argument using the Bochner formula of a slightly different function. Let

$$
g=|\nabla f|^{\frac{1}{2}} \varphi(f)
$$

with $\varphi:(0,1) \rightarrow(0, \infty)$ a $C^{\infty}$ function that will be determined later. We need to compute the Laplacian of $g$ :

$$
\begin{aligned}
\Delta g= & (\Delta h) \varphi+h \Delta \varphi+2 \nabla h \cdot \nabla \varphi \\
= & \left(-2 \rho h+h^{-1}|\nabla h|^{2}\right) \varphi+h \Delta \varphi+2 \nabla h \cdot \nabla \varphi \\
= & \left(-2 \rho h+\left(g \varphi^{-1}\right)^{-1}\left|\nabla\left(g \varphi^{-1}\right)\right|^{2}\right) \varphi+h \Delta \varphi+2 \nabla\left(g \varphi^{-1}\right) \cdot \nabla \varphi \\
= & \left(-2 \rho h+g^{-1} \varphi\left(|\nabla g|^{2} \varphi^{-2}+g^{2}\left|\nabla \varphi^{-1}\right|^{2}+2 \varphi^{-1} g \nabla \varphi^{-1} \cdot \nabla g\right)\right) \varphi \\
& +h \Delta \varphi+2 \varphi^{-1} \nabla g \cdot \nabla \varphi+2 g \nabla \varphi^{-1} \cdot \nabla \varphi
\end{aligned}
$$

and hence we get:

$$
\begin{aligned}
\Delta g= & -2 \rho g+g^{-1}|\nabla g|^{2}+g \varphi^{-2}|\nabla \varphi|^{2}-2 \varphi^{-1} \nabla \varphi \cdot \nabla g+g \varphi^{-1} \Delta \varphi \\
& +2 \varphi^{-1} \nabla g \cdot \nabla \varphi-2 g \varphi^{-2}|\nabla \varphi|^{2} \\
= & -2 \rho g+g^{-1}|\nabla g|^{2}+g \varphi^{-1} \Delta \varphi-g \varphi^{-2}|\nabla \varphi|^{2} \\
= & -2 \rho g+g^{-1}|\nabla g|^{2}+g|\nabla f|^{2}\left(\varphi^{-1} \varphi^{\prime \prime}-\varphi^{-2}\left(\varphi^{\prime}\right)^{2}\right) .
\end{aligned}
$$

Since we will use $g$ in the weighted Poincaré inequality, we need also define $\phi$, a non-negative Lipschitz function with compact support in $M$,

$$
\phi=\chi \psi,
$$


with $\psi$ depending on the $\rho$-distance function

$$
\psi(x)=\left\{\begin{array}{ccl}
1 & \text { on } & B_{\rho}(R-1) \\
R-r_{\rho} & \text { on } & B_{\rho}(R) \backslash B_{\rho}(R-1) \\
0 & \text { on } & M \backslash B_{\rho}(R)
\end{array}\right.
$$

and $\chi$ defined on the level sets of $f$ as follows

$$
\chi(x)=\left\{\begin{array}{cl}
0 & \text { on } L(0, \delta \varepsilon) \cup L(1-\delta \varepsilon, 1) \\
(-\log \delta)^{-1}(\log f-\log (\delta \varepsilon)) & \text { on } L(\delta \varepsilon, \varepsilon) \cap E \\
(-\log \delta)^{-1}(\log (1-f)-\log (\delta \varepsilon)) & \text { on } L(1-\varepsilon, 1-\delta \varepsilon) \cap F \\
1 & \text { otherwise. }
\end{array}\right.
$$

We used the notation $L(a, b)=\{x \in M \mid a \leq f(x) \leq b\}$. Let us also denote the level set of $f$ at $t$ by $l(t)=\{x \in M \mid f(x)=t\}$. In the definition of $\phi$, we fix $R, \delta$ and $\varepsilon$, and the plan is to first let $R \rightarrow \infty$ and then make $\delta$ and $\varepsilon \rightarrow 0$.

We have, since $\phi$ has compact support in $M$,

$$
\begin{aligned}
\int_{M} \phi g \nabla \phi \cdot \nabla g= & \frac{1}{4} \int_{M} \nabla \phi^{2} \cdot \nabla g^{2} \\
= & -\frac{1}{2} \int_{M} \phi^{2} g \Delta g-\frac{1}{2} \int_{M} \phi^{2}|\nabla g|^{2} \\
= & \int_{M} \phi^{2} \rho g^{2}-\int_{M} \phi^{2}|\nabla g|^{2} \\
& -\frac{1}{2} \int_{M} \phi^{2} g^{2}|\nabla f|^{2}\left(\varphi^{-1} \varphi^{\prime \prime}-\varphi^{-2}\left(\varphi^{\prime}\right)^{2}\right) .
\end{aligned}
$$

Plugging this relation into the weighted Poincaré inequality

$$
\begin{aligned}
\int_{M} \rho \phi^{2} g^{2} \leq & \int_{M}|\nabla(\phi g)|^{2}=\left(\int_{M}|\nabla \phi|^{2} g^{2}+\int_{M} \phi g \nabla \phi \cdot \nabla g\right) \\
& +\int_{M}|\nabla g|^{2} \phi^{2}+\int_{M} \phi g \nabla \phi \cdot \nabla g
\end{aligned}
$$

it follows that

$$
\frac{1}{2} \int_{M} \phi^{2} g^{2}|\nabla f|^{2}\left(\varphi^{-1} \varphi^{\prime \prime}-\varphi^{-2}\left(\varphi^{\prime}\right)^{2}\right) \leq \int_{M}|\nabla \phi|^{2} g^{2}+\int_{M} \phi g \nabla \phi \cdot \nabla g .
$$

Notice that if we define $\varphi$ by

$$
\varphi(f)=(f+1)^{-\frac{1}{2}}
$$

then $\varphi^{-1} \varphi^{\prime \prime}-\varphi^{-2}\left(\varphi^{\prime}\right)^{2}=\frac{1}{2}(f+1)^{-2}$. It is clear that the contradiction follows if we prove that the right hand side of (3) vanishes as $\phi \rightarrow 1$. Let us check this on the end $E$, the proof on the end $F=M \backslash E$ is similar. To prove our claim we follow the argument in $[\mathrm{L}-\mathrm{W}]$.

Let $E_{\rho}(R)=E \cap B_{\rho}(R)$ and denote

$$
\Omega=\left\{x \in E \mid d_{\rho}\left(x, E_{\rho}(R) \cap L(\delta \varepsilon, \varepsilon)\right) \leq 1\right\} .
$$

In the following, we will use many times the gradient estimate and decay estimate for $f$, see [L-W4]. Specifically, the following version of gradient estimate is very useful in 
our context:

$$
|\nabla f|(x) \leq C\left(\sup _{\Omega} \sqrt{\rho}\right) f(x), \text { for any } x \in L(\delta \varepsilon, \varepsilon) \cap E_{\rho}(R) .
$$

Recall also the fundamental integral decay estimate for $f$ :

$$
\int_{E_{\rho}(R) \backslash E_{\rho}(R-1)} \rho f^{2} \leq c_{1} \exp (-2 R) .
$$

Having these results on the decay of $f$, we see that

$$
\begin{aligned}
\int_{E}|\nabla \phi|^{2} g^{2} & \leq \int_{E}|\nabla \phi|^{2} h^{2} \\
& \leq 2 \int_{E}|\nabla \psi|^{2} h^{2} \chi^{2}+2 \int_{E}|\nabla \chi|^{2} h^{2} \psi^{2} \\
& \leq 2 \int_{E_{\rho}(R) \backslash E_{\rho}(R-1)} \rho|\nabla f| \chi^{2}+2(-\log \delta)^{-2} \int_{L(\delta \varepsilon, \varepsilon) \cap E}|\nabla f|^{3} f^{-2} \\
& \leq c_{2}(\delta \varepsilon)^{-1} \exp (-2 R)+c_{3}(-\log \delta)^{-1}
\end{aligned}
$$

where the last inequality follows using the Lemma 5.1 of [L-W4]. To estimate

$$
\int_{M} \phi g \nabla \phi \cdot \nabla g
$$

we use similar ideas and the fact that $\rho \rightarrow 0$ at infinity. We have, integrating by parts:

$$
\begin{aligned}
2 \int_{E} \phi g \nabla \phi \cdot \nabla g= & \frac{1}{2} \int_{E} \nabla \phi^{2} \cdot \nabla g^{2} \\
= & \frac{1}{2} \int_{L(\delta \varepsilon, \varepsilon) \cap E} \psi^{2} \nabla \chi^{2} \cdot \nabla g^{2}+\frac{1}{2} \int_{E} \chi^{2} \nabla \psi^{2} \cdot \nabla g^{2} \\
\leq & -\frac{1}{2} \int_{L(\delta \varepsilon, \varepsilon) \cap E} g^{2}\left(\psi^{2} \Delta \chi^{2}+\nabla \chi^{2} \cdot \nabla \psi^{2}\right) \\
& +\frac{1}{2} \int_{l(\varepsilon) \cap E} g^{2} \psi^{2}\left(\chi^{2}\right)_{v}-\frac{1}{2} \int_{l(\delta \varepsilon) \cap E} g^{2} \psi^{2}\left(\chi^{2}\right)_{v} \\
& +\int_{E_{\rho}(R) \backslash E_{\rho}(R-1)} \chi^{2} \psi|\nabla \psi|\left|\nabla g^{2}\right|
\end{aligned}
$$


where $v$ is the unit normal given by $|\nabla f| v=\nabla f$. The first term in the right hand side is, using that $0<\varphi<1$,

$$
\begin{gathered}
-\frac{1}{2} \int_{L(\delta \varepsilon, \varepsilon) \cap E} g^{2}\left(\psi^{2} \Delta \chi^{2}+\nabla \chi^{2} \cdot \nabla \psi^{2}\right) \leq-\int_{L(\delta \varepsilon, \varepsilon) \cap E} g^{2}\left(\psi^{2} \chi \Delta \chi+|\nabla \chi|^{2}\right) \\
+2 \int_{L(\delta \varepsilon, \varepsilon) \cap E} \chi \psi|\nabla \chi| \cdot|\nabla \psi| g^{2} \\
\leq-\int_{L(\delta \varepsilon, \varepsilon) \cap E} g^{2} \psi^{2} \chi \Delta \chi+\int_{L(\delta \varepsilon, \varepsilon) \cap E} g^{2} \chi^{2}|\nabla \psi|^{2} \\
\leq(-\log \delta)^{-1} \int_{L(\delta \varepsilon, \varepsilon) \cap E} f^{-2}|\nabla f|^{3} \varphi^{2} \chi+\int_{E}|\nabla \psi|^{2} h^{2} \chi^{2} \\
\leq c_{4} \sup _{\Omega} \sqrt{\rho}(-\log \delta)^{-1} \int_{L(\delta \varepsilon, \varepsilon) \cap E} f^{-1}|\nabla f|^{2}+c_{5}(\delta \varepsilon)^{-1} \exp (-2 R) \\
=c_{6} \sup _{\Omega} \sqrt{\rho}+c_{5}(\delta \varepsilon)^{-1} \exp (-2 R) .
\end{gathered}
$$

Note that the assumption on $\rho$ implies that $\sup _{\Omega} \sqrt{\rho} \rightarrow 0$ as $\delta, \varepsilon \rightarrow 0$. The nonzero boundary term can be bounded from above by:

$$
\begin{aligned}
\frac{1}{2} \int_{l(\varepsilon) \cap E} g^{2} \psi^{2}\left(\chi^{2}\right)_{v} & \leq \int_{l(\varepsilon) \cap E} h^{2} \chi_{v}=(-\log \delta)^{-1} \int_{l(\varepsilon) \cap E} f^{-1}|\nabla f|^{2} \\
& \leq c_{7}(-\log \delta)^{-1} .
\end{aligned}
$$

Finally, let us deal with the $|\nabla \psi|$ term:

$$
\begin{aligned}
\int_{E_{\rho}(R) \backslash E_{\rho}(R-1)} \chi^{2} \psi|\nabla \psi|\left|\nabla g^{2}\right| \leq & \int_{E_{\rho}(R) \backslash E_{\rho}(R-1)} \chi^{2} \sqrt{\rho}\left|\nabla \varphi^{2}\right| h^{2} \\
& +\int_{E_{\rho}(R) \backslash E_{\rho}(R-1)} \chi^{2} \sqrt{\rho}\left|\nabla h^{2}\right| \varphi^{2} .
\end{aligned}
$$

In the right hand side of the above formula and many times in this paper we mention $\chi^{2}$ in various integrals only as a reminder that we are also integrating on the support of $\chi$. Note that by Schwarz inequality,

$$
\begin{aligned}
\int_{E_{\rho}(R) \backslash E_{\rho}(R-1)} \chi^{2} \sqrt{\rho}\left|\nabla h^{2}\right| \varphi^{2} \leq & \left(\int_{E_{\rho}(R) \backslash E_{\rho}(R-1)} \chi^{2} \rho\right)^{\frac{1}{2}} \times \\
& \times\left(\int_{E_{\rho}(R) \backslash E_{\rho}(R-1)} \chi^{2}\left|\nabla h^{2}\right|^{2}\right)^{\frac{1}{2}} .
\end{aligned}
$$

Furthermore, we have

$$
\int_{E_{\rho}(R) \backslash E_{\rho}(R-1)} \chi^{2} \rho \leq(\delta \varepsilon)^{-2} \int_{E_{\rho}(R) \backslash E_{\rho}(R-1)} \rho f^{2} \leq(\delta \varepsilon)^{-2} \exp (-2 R) .
$$

To estimate the second term in the right hand side, one can use again the Bochner formula $\Delta h^{2} \geq-4 \rho h^{2}$, and integrate this formula with $\eta^{2} h^{2}$, where $\eta$ is a compactly supported function depending on the $\rho$-distance with support in $E_{\rho}(R+1) \backslash E_{\rho}(R-2)$ 
and $\eta=1$ on $E_{\rho}(R) \backslash E_{\rho}(R-1)$. Then, using computations similar to above it follows

$$
\int_{E_{\rho}(R) \backslash E_{\rho}(R-1)}\left|\nabla h^{2}\right|^{2} \leq c_{9} \int_{E_{\rho}(R+1) \backslash E_{\rho}(R-2)} \rho h^{4} \leq c_{10} \exp (-2 R) .
$$

Finally, observe that

$$
\int_{E_{\rho}(R) \backslash E_{\rho}(R-1)} \sqrt{\rho}\left|\nabla \varphi^{2}\right| h^{2} \leq \int_{E_{\rho}(R) \backslash E_{\rho}(R-1)} \sqrt{\rho}|\nabla f|^{2} \leq c_{11} \exp (-2 R),
$$

by Corollary 2.3 in [L-W4]. Putting everything together we see that the right hand side of (3) vanishes if we first allow $R \rightarrow \infty$, and then make $\delta, \varepsilon \rightarrow 0$. Hence $f$ has to be constant, i.e. $M$ has one nonparabolic end.

The following Corollary shows that Theorem 1 can be localized on each end of $M$. Now $\rho$ is assumed to converge to zero at infinity of only one end, but it can have any bounded behaviour on the other ends (if they exist).

Corollary 1. Let $M^{m}$ be a complete Kähler manifold of complex dimension $m \geq 2$ and with property $\left(P_{\rho}\right)$ for $\rho$ bounded. Suppose that the Ricci curvature of $M$ is bounded from below by

$$
\operatorname{Ric}_{M}(x) \geq-4 \rho(x)
$$

and that there exists a nonparabolic end $E$ where

$$
\lim _{x \rightarrow \infty, x \in E} \rho(x)=0 .
$$

Then $E$ is the only nonparabolic end of $M$.

Proof. Using the same notations as in Theorem 1, we need to show that

$$
\int_{M} \phi g \nabla \phi \cdot \nabla g \rightarrow 0
$$

for $g=\varphi|\nabla f|^{\frac{1}{2}}$. Without loss of generality assume that

$$
\inf _{x \in E} f(x)=0 \text { and } \sup _{x \in M \backslash E} f(x)=1 .
$$

Now we will choose a different $\varphi$, maintaining that $\varphi^{-1} \varphi^{\prime \prime}-\varphi^{-2}\left(\varphi^{\prime}\right)^{2}>0$. Hence let

$$
\varphi(f)=\left(f+\frac{1}{2}\right)^{-\alpha},
$$

for a fixed $\alpha>0$. Then $\varphi^{-1} \varphi^{\prime \prime}-\varphi^{-2}\left(\varphi^{\prime}\right)^{2}=\alpha\left(f+\frac{1}{2}\right)^{-2}>0$ and it is easy to see that

$$
\begin{aligned}
(-\log \delta)^{-1} \int_{L(\delta \varepsilon, \varepsilon) \cap E} f^{-2}|\nabla f|^{3} \varphi^{2} & \leq c_{1} \sup _{\Omega} \sqrt{\rho}\left(\frac{1}{2}+\delta \varepsilon\right)^{-2 \alpha}, \\
(-\log \delta)^{-1} \int_{L(1-\varepsilon, 1-\delta \varepsilon) \cap(M \backslash E)} f^{-2}|\nabla f|^{3} \varphi^{2} & \leq c_{2}\left(\frac{1}{2}+1-\varepsilon\right)^{-2 \alpha} .
\end{aligned}
$$

Therefore, using the estimates in Theorem 1 we get after letting $R \rightarrow \infty$

$$
\begin{aligned}
\frac{\alpha}{2} \int_{M} \chi^{2} g^{2}|\nabla f|^{2}\left(f+\frac{1}{2}\right)^{-2} \leq & c_{1} \sup _{\Omega} \sqrt{\rho}\left(\frac{1}{2}+\delta \varepsilon\right)^{-2 \alpha}+c_{2}\left(\frac{1}{2}+1-\varepsilon\right)^{-2 \alpha} \\
& +c_{3} 2^{2 \alpha}(-\log \delta)^{-1} .
\end{aligned}
$$


Using the assumption on $\rho$ it follows that after we let $\delta, \varepsilon \rightarrow 0$ the above inequality becomes

$$
\frac{\alpha}{2} \int_{M} g^{2}|\nabla f|^{2}\left(f+\frac{1}{2}\right)^{-2} \leq c_{2}\left(\frac{3}{2}\right)^{-2 \alpha} .
$$

However,

$$
\frac{\alpha}{2} \int_{L\left(0, \frac{1}{2}\right) \cap E} h^{2}|\nabla f|^{2}\left(f+\frac{1}{2}\right)^{-2} \leq \frac{\alpha}{2} \int_{M} g^{2}|\nabla f|^{2}\left(f+\frac{1}{2}\right)^{-2} \leq c_{2}\left(\frac{3}{2}\right)^{-2 \alpha},
$$

which is true for any $\alpha>0$. This clearly yields a contradiction if we let $\alpha \rightarrow \infty$.

Theorem 2. Let $M^{m}$ be a complete Kähler manifold of complex dimension $m \geq 3$ and with property $\left(P_{\rho}\right)$. Suppose that the bisectional curvature of $M$ is bounded from below by

If

$$
B K_{M}(x) \geq-\frac{\rho(x)}{m^{2}}
$$

then $M$ has one end.

$$
\lim _{x \rightarrow \infty} \rho(x)=0
$$

Proof. To prove Theorem 2 we exploit the same idea, though there are some particularities here. First, there is the fact that the function is not necessarily pluriharmonic, so the Bochner formula is not as simple as the one used in Theorem 1. Second, to obtain the contradiction, we will have to manipulate various integral estimates of the complex Hessian, which are more technical.

It is clear from Theorem 1 that $M$ has exactly one nonparabolic end $F$, so let us assume by absurd that $M$ has another end $E$, which has to be parabolic. Then there exists a positive harmonic function $f$ with the following properties ([L-W4, L-W]):

1. On $F$ the function satisfies the decay estimate

$$
\int_{F_{\rho}(R) \backslash F_{\rho}(R-1)} \rho f^{2} \leq c \exp (-2 R),
$$

2. On $E$ the function is proper and

3. $f(x) \rightarrow \infty$ as $x \rightarrow \infty, x \in E$ and $\inf _{F} f=0$.

Let us consider

$$
h=\left|f_{\alpha \bar{\beta}}\right|^{\frac{1}{2}}
$$

and for a smooth function $\varphi:(0, \infty) \rightarrow(0, \infty)$ let

$$
g=h \varphi(f) .
$$

Recall that under the assumption on the bisectional curvature, the following Bochner formula holds ([L-W3]):

$$
\Delta h \geq-\frac{2}{m} \rho h-\frac{m-2}{m} h^{-1}|\nabla h|^{2} .
$$

In fact, P. Li and J. Wang have proved in [L-W] that this must be an equality if $\rho \rightarrow 0$ at infinity and concluded from here that $M$ has at most two ends. The same as in the proof of Theorem 1, we can improve Li-Wang's result using $g$ as a test function instead of $h$, for a suitable choice of $\varphi$.

Now it is time to fix the cut-off functions. On the parabolic end $E$ we consider 


$$
\phi=\left\{\begin{array}{ccl}
1 & \text { on } & L(0,2 T) \cap E \\
T^{-1}(3 T-f) & \text { on } & L(2 T, 3 T) \cap E \\
0 & \text { on } & L(3 T, \infty) \cap E
\end{array},\right.
$$

because $f$ is proper on this end so there is no need of another cut-off $\psi$. On the nonparabolic end $F$ we consider the same $\phi=\chi \psi$ as in the proof of Theorem 1.

Let us compute the Laplacian of $g$ :

$$
\begin{aligned}
\Delta g= & (\Delta h) \varphi+h \Delta \varphi+2 \nabla h \cdot \nabla \varphi \\
= & \left(-\frac{2}{m} \rho h-\frac{m-2}{m} h^{-1}|\nabla h|^{2}\right) \varphi+h \Delta \varphi+2 \nabla h \cdot \nabla \varphi \\
= & \left(-\frac{2}{m} \rho g \varphi^{-1}-\frac{m-2}{m}\left(g \varphi^{-1}\right)^{-1}\left|\nabla\left(g \varphi^{-1}\right)\right|^{2}\right) \varphi \\
& +g \varphi^{-1} \Delta \varphi+2 \nabla\left(g \varphi^{-1}\right) \cdot \nabla \varphi \\
= & -\frac{2}{m} \rho g-\frac{m-2}{m} \varphi^{2} g^{-1}\left(\varphi^{-2}|\nabla g|^{2}+g^{2} \varphi^{-4}|\nabla \varphi|^{2}-2 \varphi^{-3} g \nabla \varphi \cdot \nabla g\right) \\
& +g \varphi^{-1} \Delta \varphi+2 \varphi^{-1} \nabla g \cdot \nabla \varphi-2 g \varphi^{-2}|\nabla \varphi|^{2},
\end{aligned}
$$

and therefore

$$
\begin{aligned}
\Delta g= & -\frac{2}{m} \rho g-\frac{m-2}{m} g^{-1}|\nabla g|^{2}+2 \frac{m-2}{m} \varphi^{-1} \nabla \varphi \cdot \nabla g \\
& -\frac{m-2}{m} g \varphi^{-2}|\nabla \varphi|^{2}+g \varphi^{-1} \Delta \varphi+2 \varphi^{-1} \nabla g \cdot \nabla \varphi-2 g \varphi^{-2}|\nabla \varphi|^{2} \\
= & -\frac{2}{m} \rho g-\frac{m-2}{m} g^{-1}|\nabla g|^{2}-\left(\frac{m-2}{m}+2\right) g \varphi^{-2}|\nabla \varphi|^{2} \\
& +2\left(\frac{m-2}{m}+1\right) \varphi^{-1} \nabla \varphi \cdot \nabla g+g \varphi^{-1} \Delta \varphi .
\end{aligned}
$$

Now let us use this formula to compute the following integral:

$$
\begin{gathered}
-\int_{M} \phi^{2} g \Delta g=\frac{2}{m} \int_{M} \rho g^{2} \phi^{2}+\frac{m-2}{m} \int_{M} \phi^{2}|\nabla g|^{2} \\
+\left(\frac{m-2}{m}+2\right) \int_{M} \phi^{2} g^{2} \varphi^{-2}|\nabla \varphi|^{2}+\left(\frac{m-2}{m}+1\right) \int_{M} \phi^{2} g^{2} \Delta \log \varphi \\
-\int_{M} \phi^{2} g^{2} \varphi^{-1} \Delta \varphi+\left(\frac{m-2}{m}+1\right) \int_{M} g^{2} \nabla \log \varphi \cdot \nabla \phi^{2} \\
=\frac{2}{m} \int_{M} \rho g^{2} \phi^{2}+\frac{m-2}{m} \int_{M} \phi^{2}|\nabla g|^{2} \\
+\int_{M} \phi^{2} g^{2}|\nabla f|^{2}\left(\varphi^{-2}\left(\varphi^{\prime}\right)^{2}+\frac{m-2}{m} \varphi^{-1} \varphi^{\prime \prime}\right)+\left(\frac{m-2}{m}+1\right) \int_{M} g^{2} \nabla \log \varphi \cdot \nabla \phi^{2},
\end{gathered}
$$

where we used that

$$
\begin{aligned}
-2 \int_{M} \phi^{2} g \varphi^{-1} \nabla \varphi \cdot \nabla g & =-\int_{M} \phi^{2} \nabla \log \varphi \cdot \nabla g^{2} \\
& =\int_{M} g^{2} \phi^{2} \Delta \log \varphi+\int_{M} g^{2} \nabla \log \varphi \cdot \nabla \phi^{2} .
\end{aligned}
$$


Just as in Theorem 1 the above computations are used to obtain the cross-term:

$$
\begin{aligned}
\frac{m}{2} \cdot 2 \int_{M} \phi g \nabla \phi \cdot \nabla g= & \int_{M} \rho g^{2} \phi^{2}-\int_{M} \phi^{2}|\nabla g|^{2} \\
& +\frac{m}{2} \int_{M} \phi^{2} g^{2}|\nabla f|^{2}\left(\varphi^{-2}\left(\varphi^{\prime}\right)^{2}+\frac{m-2}{m} \varphi^{-1} \varphi^{\prime \prime}\right) \\
& +(m-1) \int_{M} g^{2} \nabla \log \varphi \cdot \nabla \phi^{2}
\end{aligned}
$$

and using the weighted Poincaré inequality we get:

$$
\begin{gathered}
\frac{m}{2} \int_{M} \phi^{2} g^{2}|\nabla f|^{2} \\
\left.+-\frac{m-2}{m} \varphi^{-1} \varphi^{\prime \prime}-\varphi^{-2}\left(\varphi^{\prime}\right)^{2}\right) \leq-(m-2) \int_{M} \phi g \nabla \phi \cdot \nabla g \\
+\int_{M}|\nabla \phi|^{2} g^{2}+(m-1) \int_{M} g^{2} \nabla \log \varphi \cdot \nabla \phi^{2} .
\end{gathered}
$$

Let us now consider

$$
\varphi(f)=1-\frac{1}{f+3}
$$

It is clear that $\frac{2}{3}<\varphi<1$, and by direct calculus one can see that

$$
\begin{aligned}
-\frac{m-2}{m} \varphi^{-1} \varphi^{\prime \prime}-\varphi^{-2}\left(\varphi^{\prime}\right)^{2} & =\varphi^{-2}(f+3)^{-4}\left(2 \frac{m-2}{m}(f+2)-1\right) \\
& >\frac{1}{3} \varphi^{-2}(f+3)^{-4}
\end{aligned}
$$

This shows that if the right hand side of (4) vanishes as $\phi \rightarrow 1$ then $g$ has to be zero, i.e. $f$ is pluriharmonic. However, the same argument as in Theorem 1 will imply that $f$ must be in fact constant, which is a contradiction.

To prove the claim that the right hand side of (4) vanishes as $\phi \rightarrow 1$ we follow $[\mathrm{L}-\mathrm{W}]$. Let us work first on the end $E$ :

$$
\begin{aligned}
-\int_{E} \phi g \nabla \phi \cdot \nabla g & =\frac{1}{4} \int_{L(2 T, 3 T) \cap E} g^{2} \Delta \phi^{2}+\frac{1}{2} \int_{l(2 T) \cap E} g^{2} \phi_{v} \\
& \leq \frac{1}{2} T^{-2} \int_{L(2 T, 3 T) \cap E} g^{2}|\nabla f|^{2}+\frac{1}{2} T^{-1} \int_{l(2 T) \cap E} g^{2}|\nabla f| \\
& \leq \frac{1}{2} T^{-2} \int_{L(2 T, 3 T) \cap E} h^{2}|\nabla f|^{2}+\frac{1}{2} T^{-1} \int_{l(2 T) \cap E} h^{2}|\nabla f|
\end{aligned}
$$

We claim that there is a sequence of $T^{\prime} s$ for which both these terms converge to zero as $T \rightarrow \infty$. 
Denote $D=\left\{x \in E \mid d_{\rho}(x, L(2 T, 3 T) \cap E) \leq 1\right\}$. Note that if $f_{i j}$ denotes the real Hessian of $f$ then using Lemma 5.1 of [L-W4] we have

$$
\begin{aligned}
T^{-2} \int_{L(2 T, 3 T) \cap E} h^{2}|\nabla f|^{2} \leq & T^{-2}\left(\int_{L(2 T, 3 T) \cap E}|\nabla f|^{4}\right)^{\frac{1}{2}}\left(\int_{L(2 T, 3 T) \cap E} h^{4}\right)^{\frac{1}{2}} \\
\leq & c_{1} T^{-2}\left(\sup _{D} \sqrt{\rho}\right)\left(\int_{L(2 T, 3 T) \cap E} f^{2}|\nabla f|^{2}\right)^{\frac{1}{2}} \times \\
& \times\left(\left.\int_{L(2 T, 3 T) \cap E}\left|f_{\left.\left.\alpha \bar{\beta}\right|^{2}\right)^{\frac{1}{2}}}\right| f_{i j}\right|^{2}\right)^{\frac{1}{2}} .
\end{aligned}
$$

We now invoke the Bochner formula

$$
\Delta|\nabla f|^{2}+2 m^{-2} \rho|\nabla f|^{2} \geq 2\left|f_{i j}\right|^{2},
$$

which we integrate with a cut-off $\eta^{2}$ :

$$
2 \int_{M} \eta^{2}\left|f_{i j}\right|^{2} \leq \int_{M} \eta^{2} \Delta|\nabla f|^{2}+2 m^{-2} \int_{M} \rho \eta^{2}|\nabla f|^{2} .
$$

Integrating by parts and using that $\left|f_{i j}\right| \geq|\nabla| \nabla f||$ it results that the following inequality holds:

$$
\int_{M} \eta^{2}\left|f_{i j}\right|^{2} \leq \int_{M}|\nabla \eta|^{2}|\nabla f|^{2}+2 m^{-2} \int_{M} \rho \eta^{2}|\nabla f|^{2} .
$$

Choosing

$$
\eta=\left\{\begin{array}{ccl}
0 & \text { on } & L(0, T) \cup L(4 T, \infty) \cup F \\
T^{-1}(f-T) & \text { on } & L(T, 2 T) \cap E \\
1 & \text { on } & L(2 T, 3 T) \cap E \\
T^{-1}(4 T-f) & \text { on } & L(3 T, 4 T) \cap E,
\end{array}\right.
$$

one can see that from (5) it follows $\int_{L(2 T, 3 T) \cap E}\left|f_{i j}\right|^{2} \leq c_{3} T$. From here we deduce that

$$
\lim _{T \rightarrow \infty} T^{-2} \int_{L(2 T, 3 T) \cap E} h^{2}|\nabla f|^{2}=0,
$$

and applying the mean value theorem it results that there exists a sequence $T_{i} \rightarrow \infty$ such that

$$
\lim _{i \rightarrow \infty} T_{i}^{-1} \int_{l\left(2 T_{i}\right) \cap E} h^{2}|\nabla f|=0 .
$$

Hence, if from now on we work with $\phi$ defined by this sequence of $T_{i}$, we have $-\int_{E} \phi g \nabla \phi \cdot \nabla g \rightarrow 0$. The rest of the proof for $E$ is to notice that

$$
\int_{M} g^{2} \nabla \log \varphi \cdot \nabla \phi^{2}=-2 T^{-1} \int_{L(2 T, 3 T) \cap E} \varphi h^{2}|\nabla f|^{2}(f+3)^{-2} \phi \leq 0 .
$$

This shows that the right hand side of (4) vanishes on $E$ as $\phi \rightarrow 1$. 
On the nonparabolic end $F$ we want to take $\phi=\chi \psi$, as in Theorem 1 . We have, by computations similar to those on the parabolic end, that

$$
\begin{aligned}
-2 \int_{M} \phi g \nabla \phi \cdot \nabla g \leq & \frac{1}{2} \int_{L(\delta \varepsilon, \varepsilon) \cap F} g^{2}\left(\psi^{2} \Delta \chi^{2}+\nabla \chi^{2} \cdot \nabla \psi^{2}\right) \\
& -\frac{1}{2} \int_{l(\varepsilon) \cap F} g^{2} \psi^{2}\left(\chi^{2}\right)_{v} \\
& +\int_{F_{\rho}(R) \backslash F_{\rho}(R-1)} \chi^{2} \psi|\nabla \psi|\left|\nabla g^{2}\right| \\
\leq & \int_{L(\delta \varepsilon, \varepsilon) \cap F} g^{2} \psi^{2} \chi \Delta \chi+2 \int_{L(\delta \varepsilon, \varepsilon) \cap F} g^{2} \psi^{2}|\nabla \chi|^{2} \\
& +\int_{L(\delta \varepsilon, \varepsilon) \cap F} g^{2} \chi^{2}|\nabla \psi|^{2}+\int_{F_{\rho}(R) \backslash F_{\rho}(R-1)} \chi^{2}|\nabla \psi|\left|\nabla g^{2}\right|
\end{aligned}
$$

where we have used that

$$
-\frac{1}{2} \int_{l(\varepsilon) \cap F} g^{2} \psi^{2}\left(\chi^{2}\right)_{v}=-(\log \delta)^{-1} \int_{l(\varepsilon) \cap F} g^{2} \psi^{2} f^{-1}|\nabla f| \leq 0 .
$$

Let us denote $\Omega=\left\{x \in F \mid d_{\rho}\left(x, F_{\rho}(R) \cap L(\delta \varepsilon, \varepsilon)\right) \leq 1\right\}$. We deal with the $|\nabla \psi|$ terms first. The Schwarz inequality implies:

$$
\begin{aligned}
\int_{F_{\rho}(R) \backslash F_{\rho}(R-1)} \chi^{2}|\nabla \psi|\left|\nabla h^{2}\right| \leq & \left(\int_{F_{\rho}(R) \backslash F_{\rho}(R-1)} \chi^{2} \rho\right)^{\frac{1}{2}} \times \\
& \times\left(\int_{F_{\rho}(R) \backslash F_{\rho}(R-1)} \chi^{2}\left|\nabla h^{2}\right|^{2}\right)^{\frac{1}{2}}
\end{aligned}
$$

where now we need to use once more the Bochner formula

$$
\Delta h^{2}+\frac{4}{m} \rho h^{2} \geq \frac{4}{m}|\nabla h|^{2}
$$

to see that

$$
\int_{F_{\rho}(R) \backslash F_{\rho}(R-1)}\left|\nabla h^{2}\right|^{2} \leq c_{5} \exp (-2 R) .
$$

On the other hand,

$$
\begin{aligned}
\int_{F_{\rho}(R) \backslash F_{\rho}(R-1)} \chi^{2}|\nabla \psi|\left|\nabla g^{2}\right| \leq & \int_{L(\delta \varepsilon, \varepsilon) \cap F} \chi^{2}|\nabla \psi|\left|\nabla h^{2}\right| \varphi^{2} \\
& +\int_{F_{\rho}(R) \backslash F_{\rho}(R-1)} \chi^{2}|\nabla \psi|\left|\nabla \varphi^{2}\right| h^{2} \\
\leq & c_{6}(\delta \varepsilon)^{-1} \exp (-2 R) \\
& +\int_{F_{\rho}(R) \backslash F_{\rho}(R-1)} \sqrt{\rho}|\nabla f|\left|f_{i j}\right| \\
\leq & c_{7}(\delta \varepsilon)^{-1} \exp (-2 R) .
\end{aligned}
$$


by some estimates on $\left|f_{i j}\right|^{2}$ that follow from (5), this time with $\eta$ a cut-off depending on the $\rho$-distance function. Consequently, if we first let $R \rightarrow \infty$ and then $\delta, \varepsilon \rightarrow 0$ it follows that

$$
\int_{L(\delta \varepsilon, \varepsilon) \cap F} g^{2} \chi^{2}|\nabla \psi|^{2}+\int_{F_{\rho}(R) \backslash F_{\rho}(R-1)} \chi^{2}|\nabla \psi|\left|\nabla g^{2}\right| \rightarrow 0 .
$$

Now we need to check the $\Delta \chi$ term:

$$
\int_{L(\delta \varepsilon, \varepsilon) \cap F} g^{2} \psi^{2} \chi \Delta \chi=-(-\log \delta)^{-1} \int_{L(\delta \varepsilon, \varepsilon) \cap F} g^{2} \psi^{2} f^{-2}|\nabla f|^{2} \leq 0
$$

and the $|\nabla \chi|^{2}$ term:

$$
\begin{aligned}
\int_{L(\delta \varepsilon, \varepsilon) \cap F} g^{2} \psi^{2}|\nabla \chi|^{2} \leq & (-\log \delta)^{-2} \int_{L(\delta \varepsilon, \varepsilon) \cap F} h^{2} f^{-2}|\nabla f|^{2} \\
\leq & (-\log \delta)^{-2}\left(\int_{L(\delta \varepsilon, \varepsilon) \cap F}\left|f_{\alpha \bar{\beta}}\right|^{2} f^{-1}\right)^{\frac{1}{2}} \times \\
& \times\left(\int_{L(\delta \varepsilon, \varepsilon) \cap F} f^{-3}|\nabla f|^{4}\right)^{\frac{1}{2}} .
\end{aligned}
$$

Using integration by parts one has:

$$
\begin{aligned}
2 \int_{M} \eta^{2}\left|f_{i j}\right|^{2} f^{-1} \leq & \int_{M} \eta^{2} f^{-1}\left(\Delta|\nabla f|^{2}+2 m^{-2} \rho|\nabla f|^{2}\right) \\
\leq & 2 \int_{M} \eta^{2} f^{-2}|\nabla f|^{2}\left|f_{i j}\right|+4 \int_{M} \eta f^{-1}|\nabla f||\nabla \eta|\left|f_{i j}\right| \\
& +2 m^{-2} \int_{M} \rho \eta^{2} f^{-1}|\nabla f|^{2} \\
\leq & \frac{1}{2} \int_{M} \eta^{2} f^{-1}\left|f_{i j}\right|^{2}+2 \int_{M} \eta^{2} f^{-3}|\nabla f|^{4}+\frac{1}{2} \int_{M} \eta^{2} f^{-1}\left|f_{i j}\right|^{2} \\
& +8 \int_{M} f^{-1}|\nabla f|^{2}|\nabla \eta|^{2}+2 m^{-2} \int_{M} \rho \eta^{2} f^{-1}|\nabla f|^{2},
\end{aligned}
$$

which shows that

$$
\int_{M} \eta^{2}\left|f_{i j}\right|^{2} f^{-1} \leq 2 \int_{M} \eta^{2} f^{-3}|\nabla f|^{4}+8 \int_{M} f^{-1}|\nabla f|^{2}|\nabla \eta|^{2}+2 m^{-2} \int_{M} \rho \eta^{2} f^{-1}|\nabla f|^{2} .
$$

This holds for any compactly supported function $\eta$, in particular let us take $\eta=\eta_{1} \cdot \psi$, where

$$
\eta_{1}=\left\{\begin{array}{cll}
0 & \text { on } & L\left(0, \frac{1}{2} \delta \varepsilon\right) \cup L(2 \varepsilon, \infty) \cup E \\
(\log 2)^{-1}\left(\log f-\log \left(\frac{1}{2} \delta \varepsilon\right)\right) & \text { on } & L\left(\frac{1}{2} \delta \varepsilon, \delta \varepsilon\right) \cap F \\
1 & \text { on } & L(\delta \varepsilon, \varepsilon) \cap F \\
(\log 2)^{-1}(\log 2 \varepsilon-\log f) & \text { on } & L(\varepsilon, 2 \varepsilon) \cap F,
\end{array}\right.
$$

and $\psi$ is the cut-off defined by the $\rho$-distance function in the proof of Theorem 1 . 
One can prove now that the following estimate holds

$$
\int_{L(\delta \varepsilon, \varepsilon) \cap F}\left|f_{\alpha \bar{\beta}}\right|^{2} f^{-1} \leq \int_{L(\delta \varepsilon, \varepsilon) \cap F}\left|f_{i j}\right|^{2} f^{-1} \leq c_{8}(-\log \delta),
$$

which implies that

$$
\int_{L(\delta \varepsilon, \varepsilon) \cap F} g^{2} \psi^{2}|\nabla \chi|^{2} \rightarrow 0 \text { as } \delta, \varepsilon \rightarrow 0 .
$$

Thus, on the nonparabolic end $F$ the cross-term $-2 \int_{M} \phi g \nabla \phi \cdot \nabla g$ also vanishes as $\phi \rightarrow 1$. Finally, we have

$$
\begin{gathered}
\int_{F} g^{2} \nabla \log \varphi \cdot \nabla \phi^{2}=2(-\log \delta)^{-1} \int_{L(\delta \varepsilon, \varepsilon) \cap F} \varphi h^{2} f^{-1}|\nabla f|^{2}(f+3)^{-2} \\
\leq(-\log \delta)^{-1} \int_{L(\delta \varepsilon, \varepsilon) \cap F} h^{2} f^{-1}|\nabla f|^{2} \\
\leq(-\log \delta)^{-1}\left(\int_{L(\delta \varepsilon, \varepsilon) \cap F}\left|f_{\alpha \bar{\beta}}\right|^{2} f^{-1}\right)^{\frac{1}{2}}\left(\int_{L(\delta \varepsilon, \varepsilon) \cap F} f^{-1}|\nabla f|^{4}\right)^{\frac{1}{2}},
\end{gathered}
$$

which also converges to zero as $\varepsilon, \delta \rightarrow 0$ by the above estimates. It means that our claim is true, i.e. $g=0$. Therefore, one gets that $f_{\alpha \bar{\beta}}=0$, that is to say $f$ is pluriharmonic, contradiction. Indeed, this can be seen using the improved Bochner formula from Theorem 1 and observing that the bisectional curvature lower bound that we have assumed in Theorem 2 implies the Ricci curvature lower bound from Theorem 1. Therefore, $M$ has one end.

\section{Acknowledgements}

The author would like to express his deep gratitude to his advisor, Professor Peter $\mathrm{Li}$, for continuous guidance and support.

\section{References}

[L-T] P. Li and L.F. Tam, Harmonic functions and the structure of complete manifolds, J. Diff. Geom. 35 (1992), 359-383.

[L-W] P. Li and J. Wang, Connectedness at infinity of complete Kähler manifolds, preprint.

[L-W1] _ _ Complete manifolds with positive spectrum, J. Diff. Geom. 58 (2001), 501-534.

[L-W2] _ Complete manifolds with positive spectrum, II, J. Diff. Geom. 62 (2002), 143-162.

[L-W3] _ Comparison theorem for Kähler manifolds and positivity of spectrum, J. Diff. Geom, 69 (2005), 43-74.

[L-W4] Weighted Poincaré inequality and rigidity of complete manifolds, Ann. Scient. Éc. Norm. Sup., (4) 39 (2006), 921- 982.

[M] O. Munteanu, A sharp estimate for the bottom of the spectrum of the Laplacian on Kähler manifolds, arXiv:math.DG/0703098.

[N] M. Nakai, On Evans potential, Proc. Japan Acad. 38 (1962), 624-629.

[N-R] T. Napier and M. Ramachandran, Structure theorems for complete Kähler manifolds and applications to Lefshetz type theorems, Geom. Funct. Anal. 5 (1995), 809-851.

Department of Mathematics, University of California, Irvine, CA 92697-3875

E-mail address: omuntean@math.uci.edu 